Z. klin. Chem. u. klin. Biochem.

8. Jg., S. 339-344, Juli 1970

\title{
Bestimmung der Gesamtöstrogene in der Schwangerschaft
}

\author{
Von E. Kuss') \\ Laboratorium für Klinische Chemie und Biocbemie der I. Universitäts-Frauenklinik Mïnchen
}

(Eingegangen am 20. Januar 1970)

Es werden die verschiedenen als „Gesamtöstrogene“ bezeichneten Substanzgruppen charakterisiert. Die Methoden zur Bestimmung der Gesamtöstrogen-Ausscheidung einer Schwangeren werden definiert als Óstriol-Bestimmungen geringerer Spezifität, die dem Zweck genügen, rasch anzuzeigen, ob ein normaler oder pathologischer Zustand des Fcten wahrscheinlicher ist. Die verfügbaren Analysenmethoden werden aufgeführt, ihre Richtigkeit, Präzision und Praktikabilität werden diskutiert.

\section{Determination of the total oestrogens in pregnangy}

The different groups of substances known, collectively, as „total ocstrogens" are characterised and described. Methods for the determination of "total oestrogen" excretion in pregnancy are, in fact, oestriol measurements of low specifity, which serve to give a rapid indication of wether the foetus is probably in a normal or a pathological state. The available analytical methods are described, and their accuracy, precision and practicability are discussed.

Drei verschieden zusammengesetzte Substanzgruppen werden als „Gesamtöstrogene" bezeichnet: 1. Verbindungen, die eine gleichartige biologische Wirkung auslösen, 2. Substanzen, die durch eine analytischchemische Reaktion gemeinsam bestimmt werden können und 3. Stoffwechselprodukte, die Abkömmlinge des Östradiols-17 $\beta$ sind oder sein könnten.

$\mathrm{Zu}$ der biologisch-wirkungsmäßig definierten Substanzgruppe gehören Verbindungen, die eine als „Östrus" bezeichnete Periode des Genitalzyklus von Säugetieren induzieren. Die Testbedingungen rechtfertigen die Bezeichnung „Gesamtöstrogene“, denn es wird, zumindest im Prinzip, die gesamte den Östrus auslösende Aktivität der applizierten Probe gemessen; für die chemisch charakterisierten Substanzen ist der Begriff "Gesamtöstrogene" weniger zutreffend.

Mit den zur Zeit üblichen Methoden der chemischen „Östrogen"-Bestimmung werden Substanzen nachgewiesen, die sich in Lösungsmittel-Verteilungen wie Phenole verhalten und die beim Erhitzen mit Schwefelsäure, in der sogenannten Kober-Reaktion, eine rote, nach ITTRICH extrahierbare Farbe entwickeln. $\mathrm{Da}$ diese Verbindungen zum Teil keine östrogene Wirkung besitzen, und da der Kober-Reaktion in der chemischen "Östrogen"-Bestimmung die gleiche Funktion zukommt wie dem Östrus im biologischen Test, werden sie auch als Kober-Chromogene bezeichnet. Von Corren (1) wurden sie als KBN- bzw. KBNI-Chromogene bezeichnet, weil neben Kober (2) auch Brown (3, 4), Nocke (5) und IrTrich (6) wesentlich zur quantitativen Auswertbarkeit der Farbreaktion beigetragen haben.

Als "Gesamtöstrogene" werden schließlich auch alle Substanzen bezeichnet, die aus Östradiol-17 $\beta$ durch enzymatische Reaktionen entstanden sein könnten, un-

1) Vortrag, gehalten auf dem Symposion ,Methodik der Ostrogenbestimmungen", Köln, 5. März 1969, vor dem 15. Symposion der Deutschen Gesellschaft für Endokrinologie, Köln, 6.-8. März 1969. abhängig von ihrer tatsächlichen Biosynthese und unabhängig davon, ob die einzelne Verbindung im biologischen Test bzw. mit der Kober-Reaktion nachgewiesen werden kann. Die Östrogene im SchwangerenUrin sind größtenteils nicht tatsächliche Metabolite des Östradiols-17 $\beta$, sondern Produkte der enzymatischen Aromatisierung 16-hydroxylierter neutraler Steroide (7-9). Ihre Zusammensetzung (10-16) istin Abbildung 1 dargestellt. Obwohl nur ein Teil der Östrogene berücksichtigt wurde, ist dieses Bild aus zwei Gründen bezeichnend für die „Gesamtöstrogen"-Ausscheidung einer Schwangeren:

1. Die Menge des ausgeschiedenen Östriols ist sehr viel größer als die Menge der anderen phenolischen Steroide; vom Östriol werden täglich bis zu $50 \mathrm{mg}$ ausgeschieden, von den am stärksten vertretenen anderen Östrogenen,

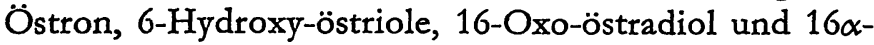
Hydroxy-östron nur etwa je 1-1,6 mg.

2. Bei der Beurteilung der Östrogen-Ausscheidung steht die Östriol-Menge im Vordergrund des Interesses, da in der Schwangerschaft die meisten Östrogen-Bestimmungen durchgeführt werden, um eine Gefährdung des Feten zu erkennen, die häufig durch eine Verminderung der Östriol-Ausscheidung der Schwangeren angezeigt wird. Die anderen Östrogene oder KoberChromogene gelten in diesem Zusammenhang z. Zt. nur als störende Begleitsubstanzen, deren Abtrennung mit erheblichem Arbeits-, Kosten- und Zeitaufwand verbunden ist. Das weist darauf hin, daß hier neben der biologischen, analytisch-chemischen und biochemischen Definition der Östrogene eine zweckgerichtete Deutung des Ausdrucks "Gesamtöstrogen-Bestimmung" berücksichtigt werden muß.

Zweck der "Gesamtöstrogen"-Bestimmung in der Schwangerschaft ist die Gewinnung einer Information über den Zustand des Feten; der Indikator dafür ist die 
von der Schwangeren in der Zeiteinheit ausgeschiedene Menge Östriol. Spezifität, Praktikabilität und Präzision der Östriol-Bestimmung müssen so gewählt werden, daß die Frage ,,ist auf Grund der Östriol-Ausscheidung ein normaler oder ein pathologischer Zustand des Feten wahrscheinlicher?" mit hinreichender Sicherheit und Schnelligkeit beantwortet werden kann.

Als Normalbereich der Östriol-Ausscheidung am Ende der Schwangerschaft werden $15-40 \mathrm{mg} /$ Tag angegegeben (Normalbereich definiert als $\bar{x} \pm 2 s \bar{x}$ ). Als Warnbereich werden Werte zwischen 12 und $8 \mathrm{mg}$, als Alarmbereich Werte zwischen 8 und $4 \mathrm{mg}$ angesehen; bei Ausscheidungen unter $4 \mathrm{mg} / \mathrm{Tag}$ in der 38.-40. Schwangerschaftswoche ist ein lebendes gesundes Kind unwahrscheinlich ${ }^{2}$ ) (17-36). Die Angaben lassen erkennen, da $ß$ eine Beeinträchtigung der Richtigkeit der Östriol-Bestimmungen um etwa $\pm 10 \%$ die klinische Bedeutung des Analysenergebnisses nicht wesentlich vermindert. Andererseits sind Schnelligkeit und Praktikabilität der Analysen von großer praktischer Bedeutung: Schnelligkeit ist geboten, damit die dem Ergebnis entsprechenden geburtshilflichen Maßnahmen rechtzeitig, eingeleitet werden können; Praktikabilität

2) Anmerkung bei der Korrektur: An der I. Universitäts-Frauenklinik München wurde unter 525 Risikoschwangerschaften $1 \mathrm{mal}$ eine Ausscheidung von weniger als $4 \mathrm{mg}$ Östrogene/24 Stdn. unmittelbar vor der Geburt eines anscheinend reifen und gesunden Kindes gefunden. Das Analysenergebnis wurde durch Kontrolle gesichert (R. GoEBEL und E. Kuss, unveröffentlicht). ist notwendig, da die Bestimmungen meist nicht von Spezialisten, sondern von den Assistentinnen gemacht werden, die auch die übrigen klinisch-chemischen Analysen durchführen. Praktikabilität ist unter diesen Umständen die entscheidende Voraussetzung dafür, $\mathrm{da} ß$ die unbedingt notwendige Präzision der Bestimmungen (Variationskoeffizient von Tag zu Tag etwa 5\%) eingehalten werden kann. Da schließlich nach Abtrennung der anderen Östrogene vom Östriol (Abb. 1) Analysenwerte erhalten werden, die nur um etwa $10 \%{ }^{3}$ ) niedriger liegen als die Ergebnisse von weniger aufwendigen Bestimmungen ohne spezifische Trennverfahren, werden von zahlreichen Laboratorien zusammen mit dem Östriol auch andere Kober-Chromogene gemessen; die Ergebnisse solcher Bestimmungen werden als „Gesamtöstrogene" angegeben. Man muß sich darüber im klaren sein, daß „Gesamtöstrogene" in diesem Sinne eine euphemistische Bezeichnung der Ergebnisse von ÖstriolBestimmungen mit geringer Spezifität ist, und daß, je nach Methode, nur ein mehr oder weniger großer Teil der anderen östrogen-wirksamen Substanzen bzw. Kober-Chromogene bzw. Östradiolmetabolite erfaßt wird.

3) Die Differenz ist u. a. davon abhängig, wie weit bei der Aufarbeitung der „Gesamtöstrogene" auch alkali-bzw. säurelabile Ostrogene erhalten bleiben und wie weit die Verbindungen mit der für Östriol optimierten Östrogenreaktion (z. B. Kober-Reaktion) erfaßt werden (l. c. 1, 41). Zur Bedeutung des Anteils der „Nicht-Östriol-Östrogene" siehe R. HobkIRK und Mitarbeiter, Clin. Chem. New York 16, 235 (1970).

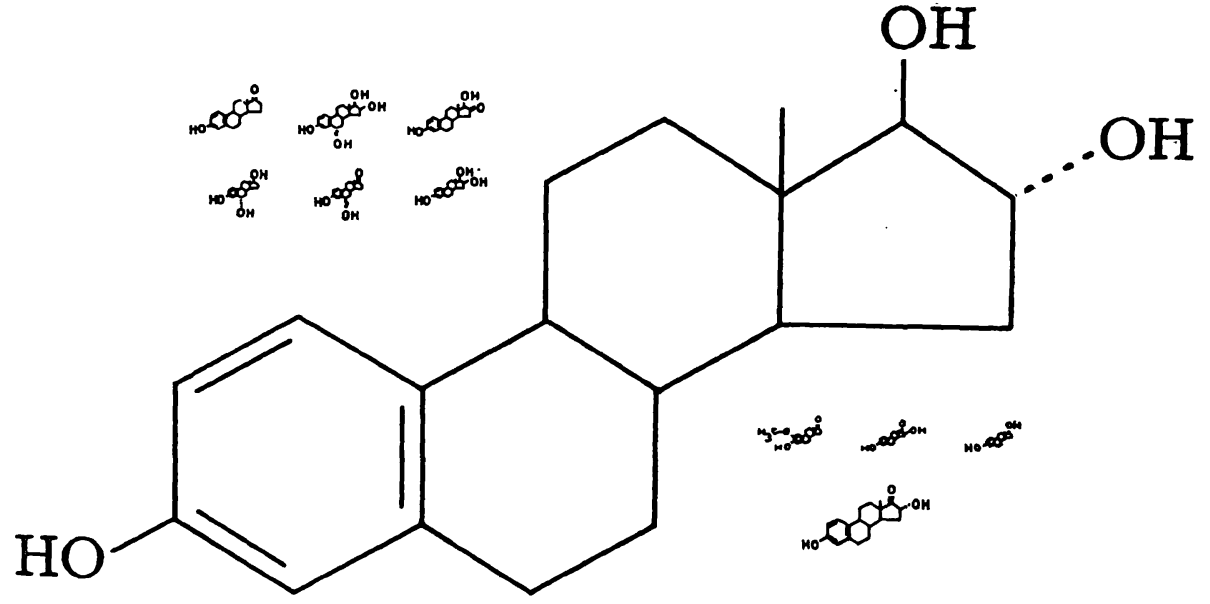

Abb. 1

Ostrogen-Ausscheidung in der Spätschwangerschaft. Dargestellt sind die Strukturformeln der Hauptbestandteile, das Größenverhältnis der Formelbilder entspricht den Literaturangaben über die Ausscheidungsmengen (1. c. 13):

$\begin{array}{lr}\text { Ostron } & 1,2 \\ \text { 6-Hydroxy-Ostriol } & 1,0 \\ \text { 2-Methoxy-Ostron } & 0,6 \\ \text { 6 } \alpha \text {-Hydroxy-Ostradiol } & 0,8 \\ \text { 6-Hydroxy-Ostron } & 0,8 \\ \text { 16epi-Ostriol } & 0,8 \\ \text { Ostriol } & 22,0 \\ \text { 16-Oxo-Ostradiol } & 1,1 \\ \text { 16 } \beta \text {-Hydroxy-Ostron } & 0,7 \\ \text { Ostradiol } & 0,4 \\ \text { 16 } \alpha \text {-Hydroxy-Ostron } & 1,6\end{array}$

Tab. 1

Präzision und Schnelligkeit von Ostrogen-Bestimmungen

\begin{tabular}{|c|c|c|c|c|}
\hline \multirow[t]{2}{*}{ Methode } & \multicolumn{2}{|c|}{ Präzision } & \multicolumn{2}{|c|}{ Schnelligkeit } \\
\hline & $\begin{array}{c}\text { Variations- } \\
\text { koeffizient (\%) }\end{array}$ & Bereich & $\begin{array}{l}\text { Probenzahl } \\
\text { pro Serie }\end{array}$ & $\begin{array}{c}\text { Zeitbedarf } \\
\text { pro Serie } \\
\text { (Stunden) }\end{array}$ \\
\hline 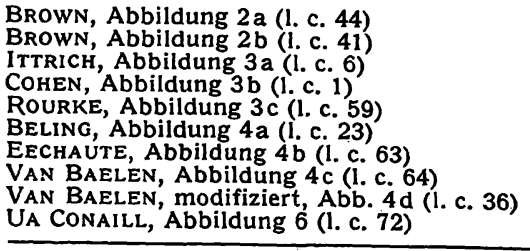 & $\begin{array}{r}15 \\
4 \\
10 \\
11 \\
6 \\
3 \\
41 . \text { c. } 36,53) \\
5 \\
6\end{array}$ & $\begin{array}{l}15 \mu \mathrm{gg} \\
1=40 \mathrm{mg} \\
2=4 \mathrm{mg} \\
3=32 \mathrm{mg} \\
0,7=23 \mathrm{mg} \\
0=40 \mu \mathrm{g} \\
0=150 \mu \mathrm{g} \\
1=22 \mathrm{mg} \\
1=30 \mathrm{mg}\end{array}$ & $\begin{array}{r}4 \\
12 \\
6 \\
6 \\
4 \\
- \\
\\
8\end{array}$ & $\begin{array}{l}48 \\
3,5 \\
3 \\
4 \\
2 \\
\frac{-}{6} \\
1\end{array}$ \\
\hline
\end{tabular}

4) Die Ergebnisse waren streng korreliert mit den nach EECHAUTE \& DEMEESTER (1. c. 63) gewonnenen Ergebnissen. 


\section{Phosphastrat alkaline}

alkalische Phosphatase

Phosphastrat acid

saure Phosphatase

Lac Dehystrat

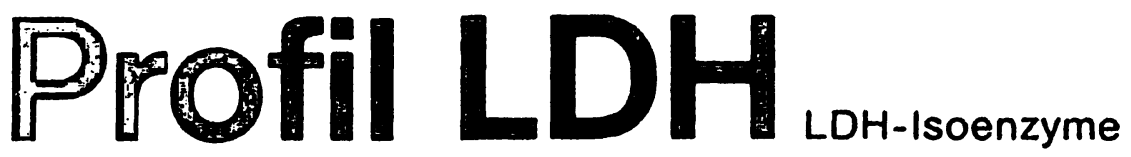

Trans $A c_{\text {oor }}^{\circ}$
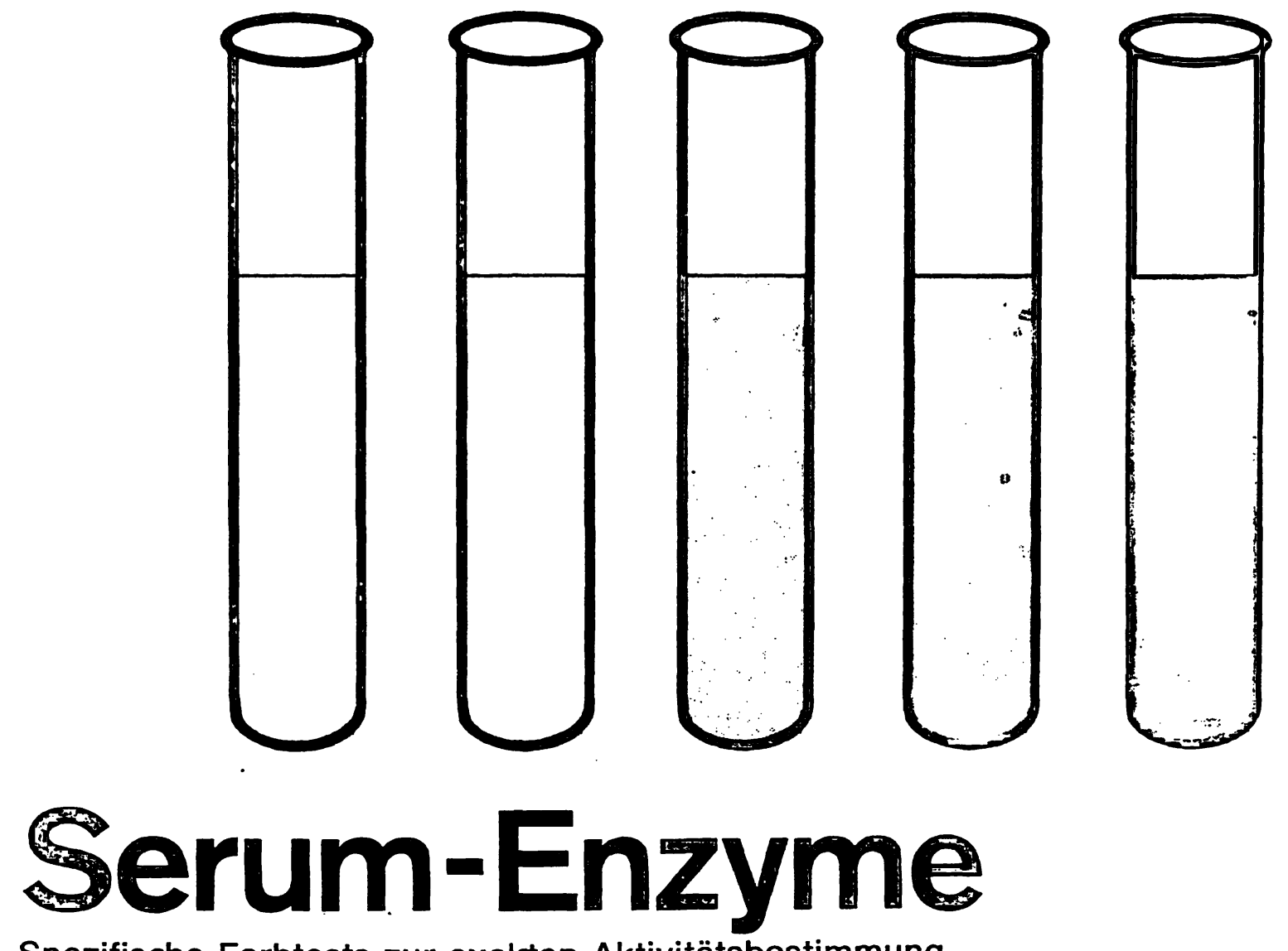

Spezifische Farbtests zur exakten Aktivitätsbestimmung

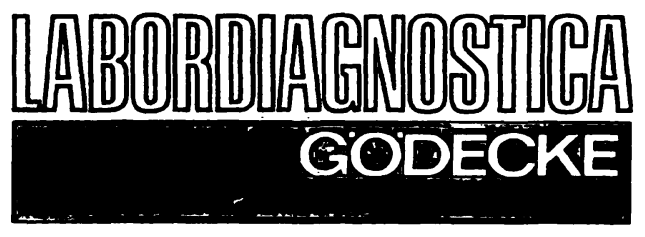

Vertrieb für Östẹrreich: Pharmazeutische Fabrik MONTAVIT GmbH.

Absam

A-6060 Solbad Hall (Tirol)
Vertrieb für die Schweiz: Cosmopharm AG $\mathrm{CH}-8040$ Zürich

Zimmerlistraße 6 


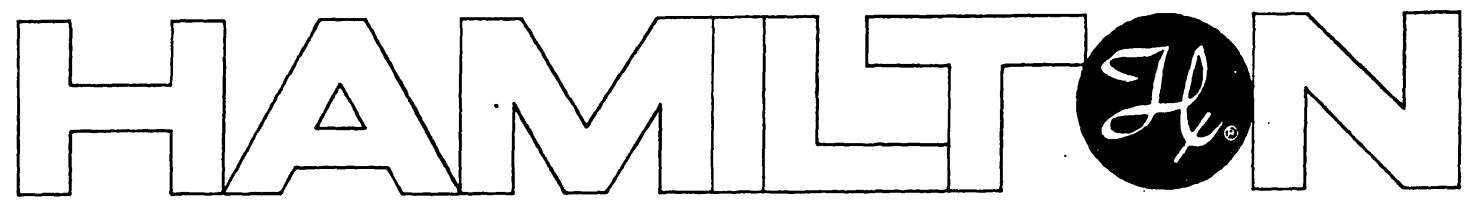

\section{Einige SPEZIALSPRITZEN zur LÖSUNG BESONDERER DOSIERPROBLEME!}

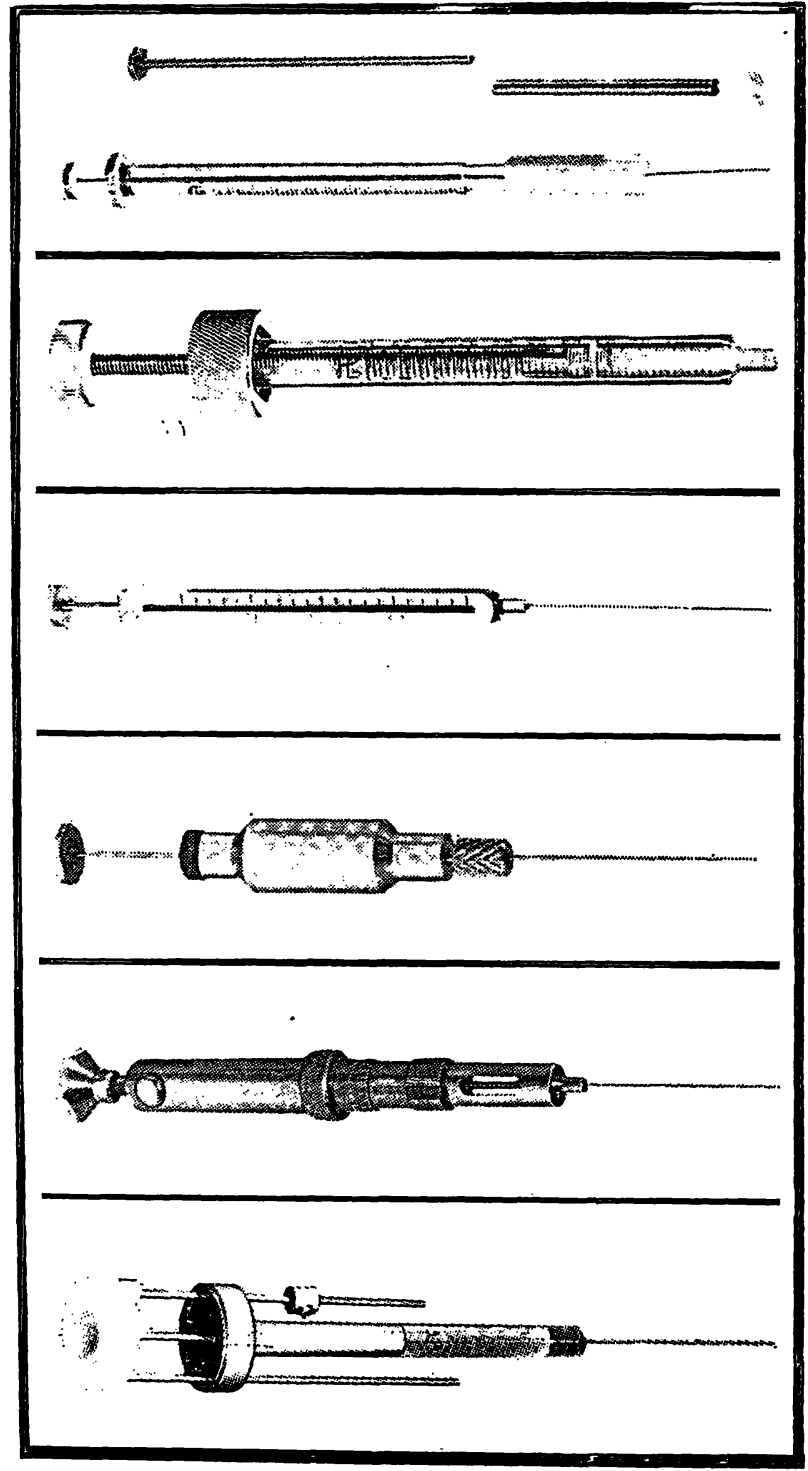

- Filterspritze, um kleinste Mengen ohne Flüssigkeitsverlust filtrieren zu können.

- Spritze mit Gewindekolben, für die kontinuierliche Abgabe von Flüssigkeiten oder Gasen.

- HS-Ganzmetallspritzen, bis zu $500^{\circ} \mathrm{C}$ erhitzbar für viskose Stoffe und Fette.

- SS 60 Probengeber,

für feste Stoffe zum Eingeben in den GC.

- CR700 Spritzen für konstante Volumen. Verstellbare aber konstante Volumenabgabe mit gleicher Einspritzgeschwindigkeit (durch Federdruck) bei gleichartigen Flüssigkeiten.

- HP 305 Hochdruck-Injektionsspritze, für Injektionen von Flüssigkeiten in Systeme mit sehr hohem Gegendruck.

Autorisierter Hăndler für die BRD:

\section{GÜTHER SGHMUDT}

2 HAMBURG 68 - POSTFACH 680104 - TEL. 6014243/6015543

Bezirksvertretungen:

Aachen: Fa. Ludwig Mohren OHG

Berlin: Fa. H. Windler KG

Bonn: Fa. C. Gerhardt

Bremen: Fa. H. Jürgens \& Co

Frankfurt: Fa. Willi Fischer \& Co.
Freiburg: Fa. Bender \& Hobein Göttingen: Fa. Bodo Schmidt Hannover: Fa. H. Jürğens \& Co. Karlsruhe: Fa. Bender \& Hobein Kiel: $\quad$ Fa. Erich Eydam

München: Fa. F.Schultheiss Münster: Fa. H. Jürgens \& Co.
Generalagentur für Europ̣a:

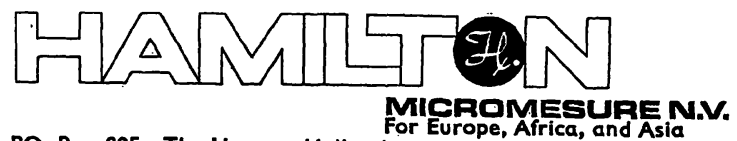

PO. Box 205 . The Hague $\cdot$ Holland 


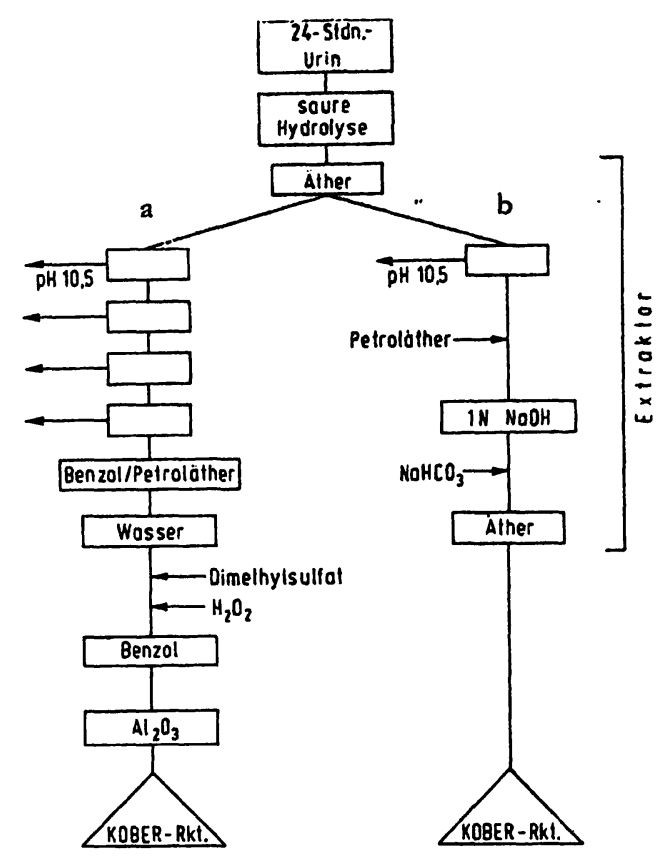

Abb. 2

Ostrogen-Bestimmungen nach BRowN

a) Bestimmung des Ostriols (1. c. 44)

b) Bestimmung der Gesamtöstrogene (1. c. 41)

Zur Gesamtöstrogen-Bestimmung aus diagnostischen Gründen wurden biologische und chemische Methoden angewendet. Der biologische Östrogen-Nachweis wurde bereits kurz nach der Entdeckung des Allen-DoisyTests zur Diagnose intakter Graviditäten herangezogen (37-39). Nach einem Hinweis von SpIELMANN und Mitarbeitern (37) hat FraNk offenbar bereits 1926 auf die Bedeutung von Östrogen-Bestimmungen bei pathologischen Schwangerschaften hingewiesen. Der positive Östrogen-Test wurde als Hinweis auf ein lebendes Kind klar abgegrenzt gegen den positiven HCG-Test nach AsChHEIM-ZoNDEK, der als Hinweis auf aktives PlazentaGewebe gedeutet wurde. Die Ergebnisse von quantitativen Gesamtöstrogen-Bestimmungen durch biologische Tests sind allerdings sehr abhängig von methodischen Einzelheiten ,so that the term oestrogenic potency becomes almost meaningless" (40). Als Alternative zur biologischen Bestimmung der Gesamtöstrogene bot sich die Bestimmung der Kober-Chromogene bzw. KBNI-Chromogene an.

In der Abbildung 2 ist eine chemische Gesamtöstrogen- bzw. Kober-Chromogen-Bestimmung skizziert und als Vergleich dazu eine Östriol-Bestimmung. Die Gesamtöstrogen-Bestimmung von Brown und Mitarbeitern (41) ist ähnlich wie die Gesamtöstrogen-Bestimmung von JAYLE und Mitarbeitern (42) und die vereinfachte Östriol-Bestimmung von BROwN und COYLE (43) eine Modifikation der 13 Jahre früher von BRown veröffentlichten Methode zur Östron-, Östradiol- und Östriol-Bestimmung, die heute noch als Standardmethode gilt. Die Tatsache, daß der gleiche Autor von einer Östriol-Bestimmung hoher Spezifität (44) über eine Östriol-Bestimmung geringerer Spezifität (43) zu einer Bestimmung der Gesamtöstrogene (41) übergegangen ist, zcigt, daß ein Fortschritt in der klinisch-chemischen Analytik nicht nur in Richtung höherer Spezifität und höherer Empfindlichkeit der Analysenmethoden gesucht wird.

Die in Abbildung $2 \mathrm{~b}$ dargestellte Gesamtöstrogen-Bestimmung unterscheidet sich von der Östriol-Bestimmung (Abb. 2a) im wesentlichen dadurch, daß auf eine Verteilung der Östrogene zwischen PetrolätherBenzol und Wasser (zur Abtrennung des Östrons und Östradiols vom Östriol) verzichtet wurde. Außerdem wurde die Chromatographie an Aluminiumoxid nach Derivatbildung (Methylierung) weggelassen. Arzawa und Pincus (45) haben bei ihrer Gesamtöstrogen-Bestimmung cine Derivatbildung (Benzoylierung) beibehalten. Vor der Hydrolyse verdünnen BROwN und Mitarbeiter (41) den Urin sehr stark mit Wasser, da Säurehydrolyse einen großen Teil des Östriols zerstört, wenn Urin mit hohem spezifischen Gewicht oder hohem Glucosegehalt eingesetzt wird (46-48). Einen anderen Ausweg bietcn die in Abbildung 3 dargestellten Verfahren, die sich dadurch auszeichnen, $\mathrm{da} ß$ die ÖstrogenKonjugate ohne Hydrolyse in die Kober-Reaktion eingebracht werden. ITrRICH (6) (Abb. 3) verzichtet in seiner "direkten" Methode nicht nur auf die Hydrolyse der Steroidkonjugate, sondern auch auf Reinigungsschritte vor der Kober-Reaktion. Mit der von ihm entdeckten Extraktion des Kober-Farbstoffes, deren Vorteile vielfach bestätigt wurden (z. B.(4)), verlegte er eine Reinigung der zu analysierenden Substanz an das Ende des Analysenganges. Einige der von ihm beschriebenen Methoden enthalten allerdings auch Arbeitsgänge wie sie von Brown (44) beschrieben wurden $(6,49)$. Die Möglichkeit, den Ittrich-Extrakt des Kober-Farbstoffes fluorimetrisch auszuwerten, wurde von ScHOLLER und Mitarbeitern (50) eingehend untersucht.

Die Zuverlässigkeit der „direkten“ ITTRICH-Methode wird dadurch beeinträchtigt, daß Urinbestandteile, besonders Gallenfarbstoffe und Pharmaka (51, 52), mit der Kober-Reaktion interferieren und unspezifiche Farbstoffe bilden oder den spezifischen Farbstoff unter-
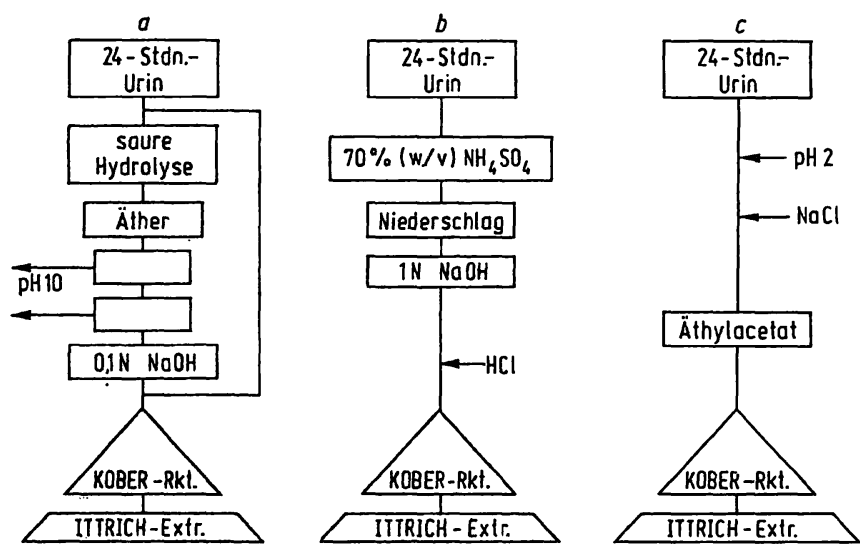

Abb. 3

Ostrogen-Bestimmungen mit Extraktionen nach ITTRICH. Bestimmung der Gesamtöstrogene

a) nach I'TTRICH (I. c. 6) und ITTRICH und SCHAETZ (1. c. 49)

b) nach COHEN (1. c. 1)

c) nach ROURKE und Mitarbeiter (1. c. 59) 
drücken. Zur Vermeidung dieser Effekte, die sich besonders bei Urinen mit geringem Gehalt an KoberChromogenen auswirken $(1,36,53)$, wurden ÖstrogenKonjugate mehr oder weniger selektiv aus dem Urin extrahiert, z. B. mit Dicyclohexylamin-acetat in Chloroform $(54,55)$ oder mit Amberlite LA 2, einem in Essigester löslichen basischen Austauscher (56), durch den aber nach GupTA und GoodwrN (57) unangenehme Emulsionen entstehen. Kürzlich wurde berichtet, daß Amberlite XAD-2, ein neutrales Polystyrol, Steroidkonjugate aus dem Urin absorbiert. Das beladene Harz wird mit Wasser gewaschen, anschließend werden die Konjugate mit Methanol eluiert (58). CoHEN (1) (Abb. 3) trennt die Östrogenkonjugate durch Ausfällen mit Ammoniumsulfat ab, RouRke und Mitarbeiter (59) (Abb. 3) durch Extraktion mit Essigester nach vorherigem Ansäuern des Urins und Aussalzen der Steroidkonjugate.

Die Gelfiltration (Abb. 4) wurde von BeLING (23) in die Östrogen-Analytik eingeführt. Nach der von ihm angegebenen Methode werden ebenfalls im ersten Schritt des Analysengangs störende Urinbestandteile abgetrennt, auch Inhibitoren der enzymatischen Hydrolyse von Steroidkonjugaten (60). In einer Modifikation dieses Verfahrens wird auf die enzymatische Hydrolyse und weitere Reinigung verzichtet und das Eluat nach der Gelfiltration (Sephadex G 15) direkt in die Kober-Reaktion eingebracht (61). Die beiden anderen in Abbildung 4 skizzierten Verfahren machen ebenfalls davon Gebrauch, daß aromatische Verbindungen an Dextrangel nicht nur wie an einem Molekularsieb fraktioniert werden (62), hier werden aber die Hydrolysate zur Gelchromatographie eingesetzt. Das große Elutionsvolumen, das freie Östrogene bei der Chromatographie nach Eechaute und Demeester (63) (Abb. 4b) benötigen, wird in der Modifikation nach VAN BAELEN und Mitarbeitern (64) (Abb. 4c) durch Verwendung von Äthylacetat als Elutionsmittel verringert.

Die Anwendung der in Abbildung 4a-c dargestellten Verfahren für die klinische Routinediagnostik ist problematisch, weil für die enzymatische Hydrolyse eine
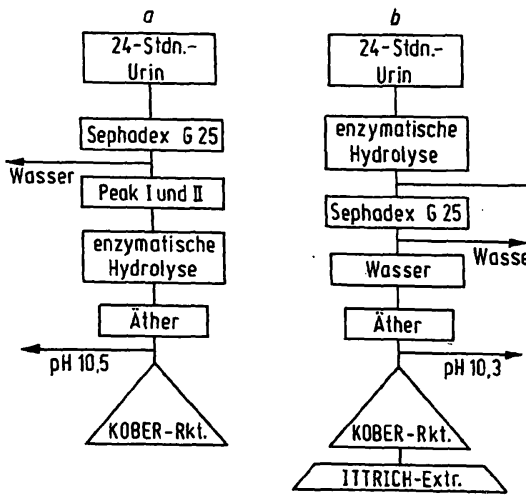

Abb. 4

Ostrogen-Bestimmungen mit Gelfiltrationen. Bestimmung a) nach BELING (1. C. 23)

b) nach Eechaute und DEMEESTER (1. c. 63)

c) nach VAN BAELEN und Mitarbeitern (1. c. 64)

d) nach VAN BAELEN (modifiziert nach 1. c. 36) lange Inkubationszeit benötigt wird (12 Stdn.); es soll allerdings auch möglich sein, bei $60^{\circ}$ in einer Stunde die Hydrolyse der Östrogen-Konjugate zu erreichen (65). Man kann aber auch ein Säurehydrolysat in die Gelfiltration nach VAN BAELEN (64) einsetzen. Dieses Verfahren hat sich bei den eigenen Untersuchungen (36) bewährt, obwohl damit wieder, die anfangs genannten Schwierigkeiten der Säurehydrolyse in Kauf genommen wurden; entsprechend den Empfehlungen anderer Autoren (41) wurde der Urin vor der Hydrolyse verdünnt.

Angaben über die Präzision $(66,67)$ und Schnelligkeit, mit der Östrogen-Bestimmungen durchgeführt wurden, sind in Tabelle 1 zusammengefaßt. Es ist zu beachten, daß die hier genannten Variationskoeffizienten aus den Ergebnissen von Doppelbestimmungen errechnet wurden und damit die Präzision der Bestimmungen in der Serie charakterisieren. Die Präzision von Tag zu Tag ist meist weniger gut, besonders wenn es sich um aufwendigere Verfahren handelt, wie z. B. die Bestimmungen von Östriol nach BRown (36).

Die von zahlreichen Autoren angegebene Richtigkeit (67) der Gesamtöstrogen-Bestimmung ist für die Routinepraxis nur bedingt gültig. Zur Charakterisierung der Richtigkeit der Methoden wurden hydrolysierten Urinproben definierte Mengen Östriol zugesetzt und ihre Wiederfindung bestimmt; sie lag meist bei $90 \%$. Bei einer Richtigkeitskontrolle im strengen Sinne müßte aber berücksichtigt werden, daß z. B. die Östriol-Zerstörung bei einer Säure-Hydrolyse je nach Urinbestandteilen von Probe zu Probe verschieden ist, da $\beta$ die Zerstörungsrate für Östriol-Konjugate anders ist als für Östriol (68) und daß außerdem noch die Zusammensetzung der Östriol-Konjugate variiert (13). Darüber hinaus wäre auch noch der Unterschied zwischen Östriol und Gesamtöstrogene zu beachten. Eine Näherungslösung bietet die Verwendung eines ,inneren Standards" für jede zu analysierende Probe. Hierzu ist wahrscheinlich das von JAFFE und LEvitz (69) verwendete Östriol-Glucosiduronat besonders geeignet. Möglicherweise könnte aber schon eine ausreichende Kontrolle der Richtigkeit aller Analysenproben erreicht werden, wenn von jeder Urinprobe eine zusätzliche Bestimmung mit Östriol als „innerem Standard" durchgeführt werden könnte. Die Voraussetzungen dafür, Analysensysteme mit hohem Probendurchsatz, wurden in letzter Zeit beschrieben. Neben dem semiautomatischen Extraktor von Brown (Abb. 2b) (41, 70) ist das AutoAnalyzer-System für Östrogen-Bestimmungen verwendet worden $(55,71,72)$. Mit der in Abbildung 6 gezeigten Anordnung werden 20 Östrogen-Bestimmungen in der Stunde durchgeführt, im Prinzip nach der Methode von ITTRICH. Die Hydrolysate werden in das System gepumpt und in den Mischspiralen mit Äther extrahiert. Im Verdampfer wird Äther abgedampft und der Rückstand mit dem Kober-Reagenz erhitzt. Der Kober-Farbstoff wird anschließend mit dem Ittrich-Reagenz extrahiert und im Photometer bei $540 \mathrm{~nm}$ und, zur Korrektur des Untergrunds, bei $420 \mathrm{~nm}$ gemessen. 


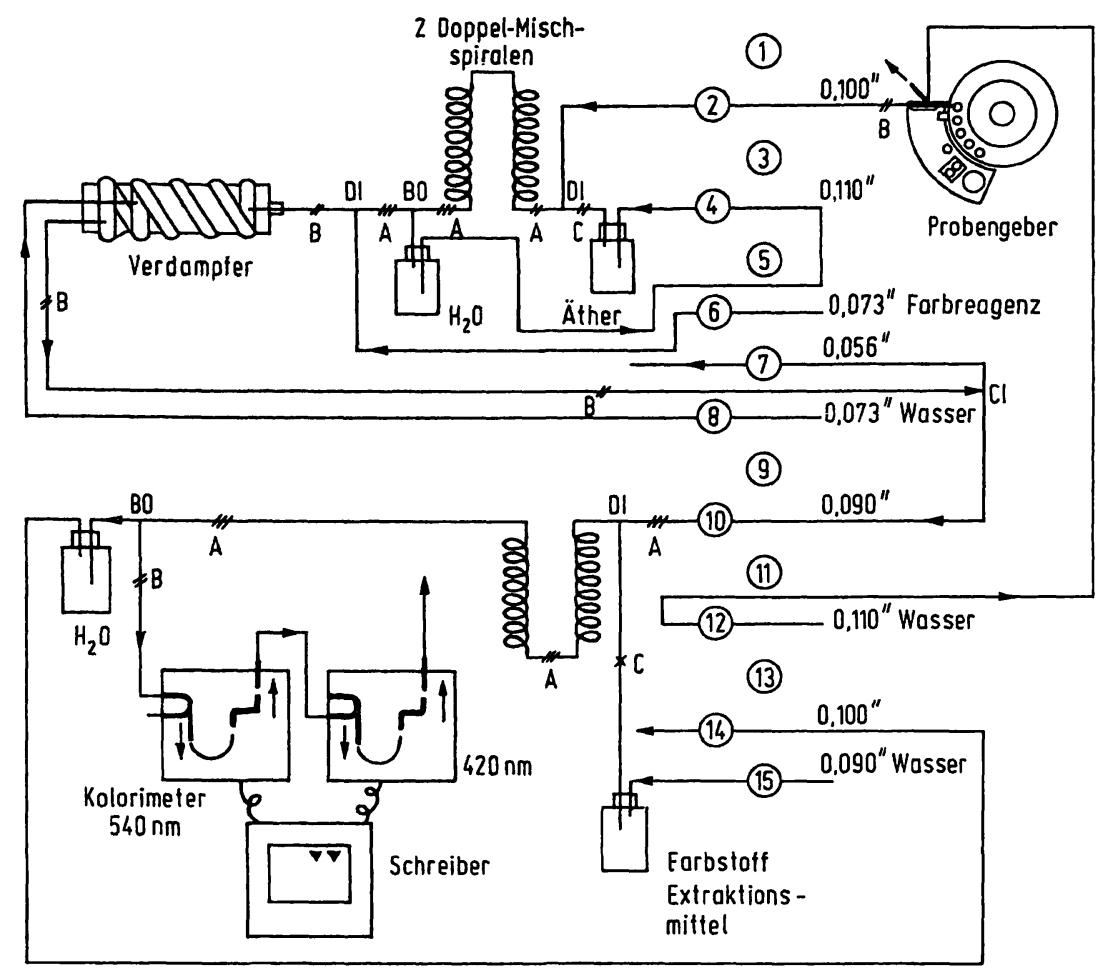

Die Diagnose einer durch verminderte Östriol-Ausscheidung gekennzeichneten fetoplazentaren Dysfunktion soll für die Geburtshilfe von ebenso großer Bedeutung sein wie die Diagnose einer BlutgruppenInkompatibilität oder einer Zervix-Insuffizienz (73). Die Kosten der dazu notwendigen Untersuchungen wurden berechnet (74): nur etwa 50-160£ (500-1500 DM) wurden für Östrogen-Bestimmungen bei Risikoschwangerschaften ausgegeben, damit ein Kind auf Grund der hierdurch induzierten Maßnahmen am Leben erhalten werden konnte. Die Vielfalt der Methoden zur ÖstrogenBestimmung in der Spätschwangerschaft, von denen die Gruppe der „Gesamtöstrogen"-Bestimmungen hier dargestellt wurde, ermöglicht es jeder Klinik, ein ihrem Rahmen angemessenes Verfahren anzuwenden. Daß bei den eigenen Analysen die Kober-Reaktion bevorzugt wird, mag in der Tradition der I. Universitäts-Frauenklink München begründet sein: nach WIELAND (75) lieferte sie vor 40 Jahren das Ausgangsmaterial für die ersten Kober-Reaktionen von Östrogenen.

\section{Literatur}

1. Cohen, S. L., J. Clin. Endocr. Springfield 26, 994 (1966). 2. Kober, S., Biochem. Z. 239, 209 (1931). - 3. Brown, J. B., J. Endocrinology 8, 196 (1952). - 4. Brown, J. B., C. MACNaughtan, M. A. Smith und B. S MYTH, J. Endoctinology 40 , 175 (1968). - 5. Nocke, W., Biochem. J. 78, 593 (1961). 6. ItTRICH, G., Acta endocr. Kopenhagen 35, 34 (1960). - 7. Cassmer, O., Acta endocr. Kopenhagen, Suppl. 45 (1959). 8. Davis, M. E., M. WIENER, H. I. JACOBSON und E. V. JENSEN, Amer. J. Obstet. Gynec. 87, 979 (1963). -9. НовKIRK, R. und M. Nilsen, J. Clin. Endocr., Springfield, 26, 625 (1966). - 10. HobkIRK, R. und M. Nirsen, J. Clin. Endocr., Springfield, 22, 142 (1962). - 11. Hobkirk, R. und M. NILSEN, J. Clin. Endocr., Springfield, 22, 134 (1962). - 12. HOBKIRK, R. und M. NiLSEN, J. Clin. Endocr., Springfield, 23, 274 (1963). - 13. Breuer, H., in: Cassano, C., Research on Steroids, Vol. 1, ,Il Pensiero Scientifico", Rom (1964). - 14. Hähnet, R. und M. G. B. A. RahmaN, Clin chim. Acta, Amsterdam 13, 797 (1966). - 15. Härnel, R. und M. G. B. A. RaHMAN, Biochem. J. 105, 1047 (1967). - 16. SMIth, E. R. und A. E. Kelire, Biochem. J. 104, 83 (1967). 17. Zondek, B. und S. Goldoberg, J. Obstett. Gynaec. Brit. Empire 64, 1 (1957). - 18. KAISER, R., Arch. Gynäk. 192, 428 (1960). - 19. Furuhjelm, M., Acta obstetr. gynec. Scand. 41, 370 (1962). - 20. Greene, J. W., J. C. Touchstone und H. Fields, Amer. J. Med. Sc. 244, 756 (1962). - 21. Greene, J. W. und J. C. Touchstone, Ámer. J. Obstetr. Gynec. 85, 1 (1963). 22. Greene, J. W., K. Smith, G. C. KyLE, J. C. Touchstone und J. L. Duhring, Amer. J. Obstetr. Gynec. 91, 684 (1965). -
23. Beling, C. G., Acta endocr. Kopenhagen, 79 (1963) Suppl. 24. Frandsen, V. A. und G. Stakeman, Acta endoct. Kopenhagen, 44, 183 (1963). - 25. KLOPPER, A. und R. STEPHENSEN, J. Obstetr. Gynaec. Brit. Commonw. 73, 282 (1966). - 26. Strand, A., Acta obstetr. gynec. Scand. 45, 125 (1966) Suppl. 1. 27. Wallace, S. J. und E. A. MrCrire, Lancet, London 1966/II, 560. - 28. Hausknecht, R. U., Obstetr. Gynec. 30, 639 (1967). 29. Mrchie, E. A., J. Obstetr. Gynaec. Brit. Commonw. 74, 896 (1967). - 30. Acevedo, H. F., H. S. Strickler, J. Gilmore, B. A. Vela, E. A. Campell und B. J. Arras, Amer. J. Obstetr. Gynec. 102, 867 (1968). - 31. BeIscher, N. A., J. B. Brown und M. A. Smrtr, J. Obstetr. Gynaec. Brit. Commonw. 75, 622 (1968). - 32. Corson, St. L. und R. J. Bolognese, Amer. J. Obstetr. Gynec. 101, 633 (1968). - 33. Magedantz, H. G., D. Klausner, K. R. Ryan und S. S. C. Yen, Obstetr. Gynec. 32, 610 (1968). - 34. Neill, D. W. und C. A. J. MaCafee, J. Obstetr. Gynaec. Brit. Commonw. 75, 172 (1968). - 35. OAKEY, R. E., S. R. Strtcir, R. F. Heys und J. S. Scort: Lancet, London 1968/I, 331. - 36. Goeber, R., K. WrnkLeR und E. Kuss, 15. Symposion der Deutschen Gesellschaft für Endokrinologie (1969). - 37. Spizlman, F., M. A. Goldberger und R. T. Frank, J. Amer. Med. Ass. 101, 266 (1933). - 38. SMTth, G. V. und O. W. SMITH, Proc. Soc. exper. Biol. Med., N. Y. 30, 918 (1933). - 39. Smrth, G. V. und O. W. Smith, Physiol. Rev., Baltimore 28, 1 (1948). - 40. O'Donnell, V. J. und J. R. K. Preedy, in: Gray, C. H. and A.L. BACHARACH, Hormones in Blood, Academic Press, London and New York (1961). - 41. Brown, J. B., 
S. C. MacLeod, C. MacNaughtan, M. A. Smith und B. Smyth, J. Endocrinology 42, 5 (1968). - 42. JAYLE, M. F., R. SCHOLLER, M. Heron und S. Metay, Clin. chim. acta (Amsterdam) 4, 276 (1959). - 43. Brown, J. B. und M. G. Coyle, J. Obstetr. Gynaec. Brit. Commonw. 70, 219 (1963). - 44. Brown, J. B., Biochem. J. 60, 185 (1955). - 45. Aizawa, Y., und G. Pincus, Steroids 4, 249 (1964). - 46. Brown, J. B. und H. A. F. BlaIR, J. Endocrinology 17, 411 (1958). - 47. Yousem, H. L., D. Solomion und D. Strummer, Amer. J. Obstetr. Gynec. 95, 595 (1966). 48. Schindler, A. E., V. Ratanasopa und W. L. Herrman, Clin. Chem. (New York) 13, 186 (1967). - 49. ItrRICH, G. und Schaetz, Zbl. Gynäk. 89, 505 (1967). - 50. Scholler, R., P. Leymarie, M. Heron und M. F. Jayle, Acta endocr. Kopenhagen 107 (1966) Suppl. - 51. Adlercreutz, H. und K.-O. Schauman, Acta endocr. Kopenhagen 46, 230 (1964). - 52. Sele, V. und V. A. Frandsen, Acta obstetr. gynec. Scand. 46, Suppl. 9, 43 (1967). - 53. Breuer, H. und J. Gertz, Clin. chim. Acta (Amsterdam) 5, 544 (1960). - 54. Beall, D. und G. A. Grant, Chemistry in Canada 2, 129 (1950). - 55. Fournier, A., T. W. Shields, R. P. Neil, C. M. Hayes und Papineau-Couture, Technicon Symposium "Automation in Analytical Chemistry“. New York (1966). - 56. Kushinsky, S. und J. TANG, Acta endocr. Kopenhagen, 43, 345 (1963). - 57. GuptA, D. und J. Goodwin, Steroids 8, 195 (1966). - 58. Bradiow, H. L.,
Steroids 11, 265 (1968). - 59. Rourke, J. E., L. D. MArshall und) T. F. SHeltey, Amer. J. Obstetr. Gynec. 100, 331 (1968). 60. AdlerCrRutz, H., Acta endocr., Kopenhagen 57, 49 (1968). 61. Kreutzer, H. J. H. und P. N. Meulendijk, Clin chim. Acta (Amsterdam) 22, 481 (1968). - 62. Gelotre, B., J. Chromatogr. 3, 330 (1960). - 63. Eechaute, W. und G. Demeester, J. Clin. Endocr., Springfield 25, 480 (1965). - 64. VAN BaELEN, H., W. Heyns und P. DE MOOR, J. Clin. Endocr., Springfield 27, 1056 (1967). - 65. Scholler, R.;' S. Metay, S. Herbin und M. F. JAYLE, Europ. J. Steroids 1, 373 (1966). - 66. BorTH, R., Ciba Foundation Colloquia on Endocrinology 2, 45 (1952). 67. Bürtner, H. und D. Stamm, diese Z. 4, 303 (1966). 68. Katzman, Ph. A., R. F. Straw, H. J. Buehler und E. A. Dorsx, Recent Progr. Hormone Res., N. Y. 9, 45 (1954). 69. JAFFe, St. H. und M. Levitz, Amer. J. Obstetr. Gynec. 98, 992 (1967). - 70. SMYTH, Barbara J., Symposion „Methodik der Östrogenbestimmungen", 5. März 1969 in Köln. - 71. STrICKLER, H. S., S. S. Holt, H. F. Acevedo, E. SaIER und R. C. Grauer, Steroids 9, 193 (1967). - 72. UA ConaILl, D. und G. G. MuIr, Clin. Chem. (New York) 14, 1010 (1968). - 73. GARDNER, H. L., Amer. J. Obstetr. Gynec. 91, 690 (1965). - 74. Heys, R. F., J.S. Scotr, R. E. OAKEY und S. R. StrTCH, Lancet, London 1968/I, 328. - 75. Wreland, H., W. Straub und Th. DorfMüLLER, Hoppe-Seylers Z. physiol. Chem. 186, 97 (1929).

Priv.-Doz, Dr. Dr. E. Kuss, I. Frauenklinik der Universität München D-8 München 15, Maistraße 11 